\title{
CORRESPONDENCE Response to Park et al.
}

Genetics in Medicine (2021) 23:1173-1174; https://doi.org/10.1038/ s41436-021-01105-0

Park et al. ${ }^{1}$ reports in this work on the screening of diagnostic exomes for rare heterozygous STUB1 variants in 7,832 cases, including 847 patients with ataxia. This is the largest screening of STUB1 variants published so far, since our own study. ${ }^{2}$ The authors did not indicate the origin of the cases screened, the conditions of sampling, nor the project(s) from which they were included, making the assessment of potential screening bias difficult.

Rare heterozygous variants were detected in 46 of 847 ataxia patients $(5.5 \%)$ in their series. Interestingly, this rate is close to the $7 \%$ of heterozygous variants detected in our cohort. ${ }^{2}$ Altogether, the two studies confirm undoubtedly that heterozygous variants in STUB1 are a frequent cause in ataxia leading to SCA48. This reinforces the need to consider STUB1 in the systematic analysis of data coming from genome/exome sequencing. Alternatively, this gene could be included in panels as a major causal gene in non-polyQ spinocerebellar ataxias (SCAs), even if the interpretation of some variants can be challenging as discussed below.

Also in line with what has been observed by us and others, ${ }^{2-5}$ cognitive impairment was predominant in STUB1 related ataxia in 14 patients, but there is no indication on how this was tested and for how many of the 847 ataxia patients. In our observations, this rate was up to $50 \%$. This high rate may be due to more extensive neuropsychological testing including affected relatives as well.

Incomplete penetrance and variability of the age at onset, ranging from the first decade of life to $>70$ years of age could indeed complicate genetic counseling. Strikingly, Park et al. describe two healthy relatives who carry a frameshift variant presumed to have a higher and less questionable pathogenicity. Do the authors know if these patients have been re-evaluated since? In the cohort reported here, 16 of 46 patients were harboring protein-truncating variants of STUB1 as well as three individuals in the nonataxia cohort.

Furthermore, the authors suggested that the variation p.Arg222Lys, detected in a familial case from our series, ${ }^{1}$ and found in four individuals from the same nonataxic cohort, was unlikely to be pathogenic. Having three or four heterozygotes in a large, but unfollowed, cohort used as controls may not be sufficient to respectively consider or exclude a rare variant as a potential pathogenic variant. The identification of nonataxic patients with protein-truncating variants in STUB1 in their study with nearly the same low frequency, and the fact that proteintruncating variants in STUB1 are present in the gnomAD database (https://gnomad.broadinstitute.org/), again at a very low frequency, did not lead us to exclude all protein-truncating variants as causal, but rather confirm the variable penetrance of STUB1 variants. This is especially true for the recurrent $p$. Tyr49Cys independently detected in patients by us and Park et al. This variant is also present in 3/67,442 individuals from the non-neuro cohort in gnomAD v3.1 although its role in the disease was confirmed. This illustrates the difficulty in only referring to minor allele frequencies in public databases to filter variants, as one cannot exclude that very few individuals are carrying variants with variable penetrance, or may not have developed any pathology at the time of their inclusion as controls. Unfortunately, segregation analysis of the p.Arg222Lys was not possible in our study. Nonetheless, we systematically checked for the segregation of candidate variants to further support the list of STUB1 variants presented in our paper in nearly half of the families $(13 / 30),{ }^{2}$ showing the high added value of segregation analyses in addition to pathogenicity prediction tools.

A majority of patients from our series were women (70\%), in contrast with the frequency of affected women found by Park et al. (43\%). In addition, segregation analyses revealed that almost all variants were transmitted by affected mothers. ${ }^{2}$ We cannot exclude that this ratio may have been incorrectly estimated given the small number of patients. It would be interesting first to know the sex ratio in their starting cohort of patients with ataxia and second, to what extent the segregation analysis was performed in their own cohort. Potential new cases or new healthy heterozygotes could be revealed this way, bringing forth important information about the variable penetrance of STUB1 variants. Nevertheless, we agree that sex-dependent penetrance of STUB1 variants observed in our series but not replicated here needs to be further explored.

Lastly, the authors mentioned that they detected rare STUB1 variants in 65 individuals with "unrelated phenotypes." It would have been very interesting to have a precise description of those phenotypes, since it could expand the clinical spectrum of STUB1 variants.

Since the first description of heterozygous STUB1 variations leading to SCA48, the role of this gene in ataxia has already been expanded. ${ }^{2-5}$ Conclusions from the study from Park et al. largely support that STUB1 variants are a frequent cause of ataxia with predominant cognitive impairment. The other side of the coin is that clinicians will be confronted more frequently with the clinical interpretation of STUB1 variants. A comprehensive unified database of these variants may be helpful in this context. We fully agree with the authors about the usefulness of developing functional assays, to improve knowledge about the pathophysiological role of this gene.

Mathieu Barbier ${ }^{1}$, Claire Sophie Davoine ${ }^{1}$, Alexis Brice ${ }^{1}$ and Alexandra Durr iD ${ }^{1 凶}$

${ }^{1}$ Sorbonne Université, Institut du Cerveau-Paris Brain Institute (ICM), AP-HP, INSERM, CNRS, University Hospital Pitié-Salpêtrière, Paris, France. ${ }^{\bowtie}$ email: alexandra.durr@icm-institute.org

Received: 2 December 2020; Revised: 16 December 2020; Accepted: 12 January 2021;

Published online: 24 February 2021

\section{REFERENCES}

1. Park, J. et al. Challenges in interpreting heterozygous STUB1 variants. Genet. Med. (in press)

2. Roux, T. et al. Clinical, neuropathological, and genetic characterization of STUB1 variants in cerebellar ataxias: a frequent cause of predominant cognitive impairment. Genet. Med. 22, 1851-1862, https://doi.org/10.1038/s41436-020-0899-x (2020). 
1174

3. Genis, D. et al. Heterozygous STUB1 mutation causes familial ataxia with cognitive affective syndrome (SCA48). Neurology. 91, e1988, https://doi.org/10.1212/ WNL.0000000000006550 (2018).

4. De Michele, G. et al. Spinocerebellar ataxia 48 presenting with ataxia associated with cognitive, psychiatric, and extrapyramidal features: A report of two Italian families. Parkinsonism Relat. Disord. 65, 91-96, https://doi.org/10.1016/j. parkreldis.2019.05.001 (2019).

5. Lieto, M. et al. The complex phenotype of spinocerebellar ataxia type 48 in eight unrelated Italian families. Eur. J. Neurol. 27, 498-505, https://doi.org/10.1111/ ene.14094 (2020)

\section{COMPETING INTERESTS}

The authors declare no competing interests.

\section{ADDITIONAL INFORMATION}

Correspondence and requests for materials should be addressed to A.D.

Reprints and permission information is available at http://www.nature.com/ reprints
Publisher's note Springer Nature remains neutral with regard to jurisdictional claims in published maps and institutional affiliations.

Open Access This article is licensed under a Creative Commons BY NC SA Attribution-NonCommercial-ShareAlike 4.0 International License, which permits any non-commercial use, sharing, adaptation, distribution and reproduction in any medium or format, as long as you give appropriate credit to the original author(s) and the source, provide a link to the Creative Commons license, and indicate if changes were made. If you remix, transform, or build upon this article or a part thereof, you must distribute your contributions under the same license as the original. The images or other third party material in this article are included in the article's Creative Commons license, unless indicated otherwise in a credit line to the material. If material is not included in the article's Creative Commons license and your intended use is not permitted by statutory regulation or exceeds the permitted use, you will need to obtain permission directly from the copyright holder. To view a copy of this license, visit http://creativecommons.org/licenses/by-nc-sa/4.0/.

C The Author(s) 2021 